\title{
Accessing High Spatial Resolution Low-Loss EELS Information without Čerenkov Radiation
}

Julia I. Deitz, ${ }^{1}$, Tyler J. Grassman ${ }^{1,2}$, David W. McComb, ${ }^{1}$

${ }^{1}$ Dept. of Materials Science \& Engineering, The Ohio State University, Columbus, OH, 43210, USA

${ }^{2}$ Dept. of Electrical \& Computer Engineering, The Ohio State University, Columbus, OH, 43210, USA

Advances in monochromation for scanning transmission electron microscopy (STEM) uniquely position electron energy-loss spectroscopy (EELS) to be the only analytical technique that can provide atomically-resolved chemical and electronic structure information. These advances open the potential for the detailed investigation of bandgaps and defect levels using the low energy-loss region of the spectrum, which is critical for continued progress in the development of advanced semiconductor materials and structures. However, Čerenkov radiation in the low energy-loss region has thus far limited the accuracy of such measurements. In this contribution, we report on EELS investigations carried out using an accelerating voltage of $40 \mathrm{kV}$ in monochromated STEM, allowing for the determination of electronic structure information for semiconductors with high spatial resolution.

For semiconductors studied under typical accelerating voltages used in STEM work, the speed of electrons travelling through the specimen, $v$, will surpass the phase velocity of light within the medium of the specimen, $v_{p}$. The condition for emission of Čerenkov radiation is thus defined by the criterion $v>$ $v_{p}=c_{0} / n(E)$ where $c_{0}$ is the speed of light, and $n(E)$ is energy (or wavelength) dependent refractive index of the material [1]. The signal due to Cerenkov losses that will appear in an EELS spectrum will depend on sample thickness, collection semi-angle (since Čerenkov radiation is highly forward scattered), and most significantly, accelerating voltage [2]. Figure 1 details the refractive index as a function of accelerating voltage necessary to meet the condition for Čerenkov radiation at constant thickness and collection semi-angle. Given that most semiconductors have refractive indexes above 2.5, Čerenkov losses will affect their energy-loss spectra at most accelerating voltages, leading to inaccurate values for the complex dielectric function and inaccurate interpretation of electronic structure.

Figure 2 illustrates single scattering distributions for $\mathrm{Si}$ at $300 \mathrm{kV}$ and $60 \mathrm{kV}$ to demonstrate the impact of Čerenkov losses on EELS interpretation. At $300 \mathrm{kV}$, there is a significant peak around $3 \mathrm{eV}$ that is almost unobservable at $60 \mathrm{kV}$, due to Čerenkov radiation. To avoid the impact of Čerenkov losses on EELS interpretation for semiconductors, it is necessary to use lower accelerating voltages. The Ohio State University's Center for Electron Microscopy and Analysis has recently aligned the image-corrected Titan $^{\text {TM }}$ G2 STEM to operate at $40 \mathrm{kV}$, meaning that it is possible to avoid the impact of Čerenkov losses entirely for most semiconductors, while still achieving high spatial resolution. In this study, the impacts of Čerenkov losses at $300 \mathrm{kV}, 60 \mathrm{kV}$, and $40 \mathrm{kV}$ are investigated for a GaP/Si heterostructure with varying values of collection semi-angle. Comparisons of the spectra at varied accelerating voltages are made for both the $\mathrm{GaP}$ and $\mathrm{Si}$ regions of the sample to further verify the role of the material type on the prevalence of Čerenkov radiation. Experimental data will be complimented with simulations of energy-loss spectra, with and without Čerenkov losses, calculated via the Kroger equation [3]. Čerenkov radiation is shown to dominate at $300 \mathrm{kV}$, but to be nearly completely removed at $60 \mathrm{kV}$ and $40 \mathrm{kV}$. The ability to obtain high spatial resolution EELS information without the altering effects of Cerenkov radiation means that it is possible to accurately study semiconductor electronic structure details at the atomic scale, allowing for unprecedented access to defect and interfacial investigation. 
[1] R. F. Egerton, Electron energy-loss spectroscopy in the electron microscope, 2nd Edition, Plenum press: New York (1996)

[2] E. Kröger, Z. Phys. 235, 403 (1970)

[3] M. Stoger-Pollach, H. Franco, P. Schattschneider, S. Lazar, B. Schaffer, W. Grogger and H. W.

Zandbergen, Micron, 37, 396 (2006).

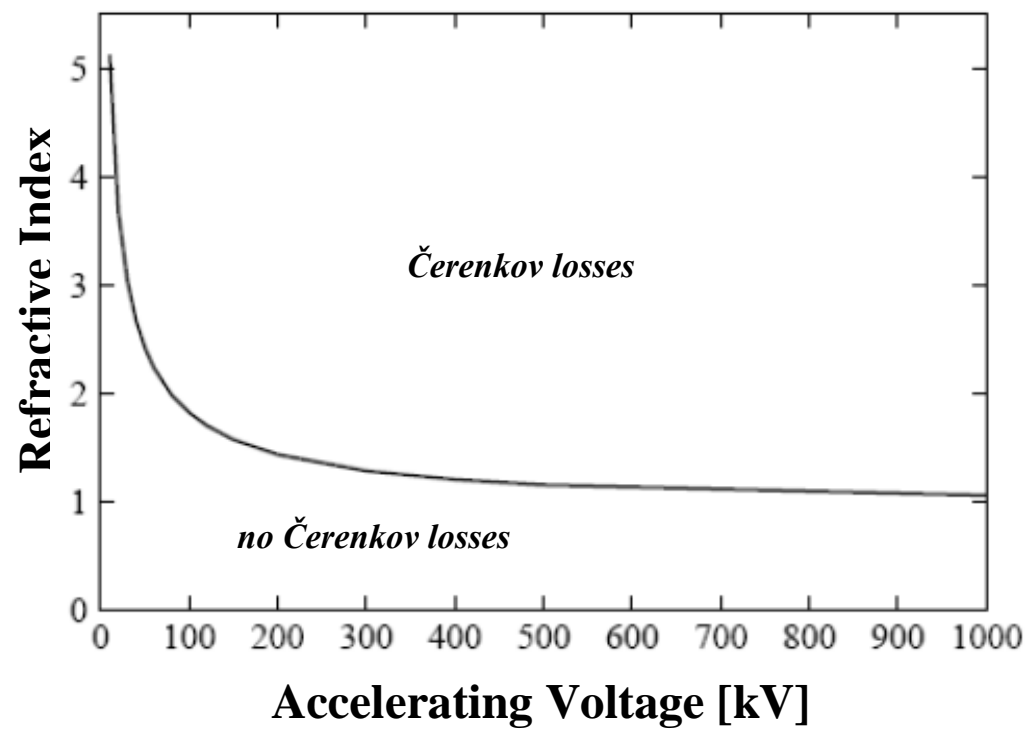

Figure 1. Refractive index criteria for meeting the conditions for Čerenkov losses as a function of accelerating voltage. Ref [3]

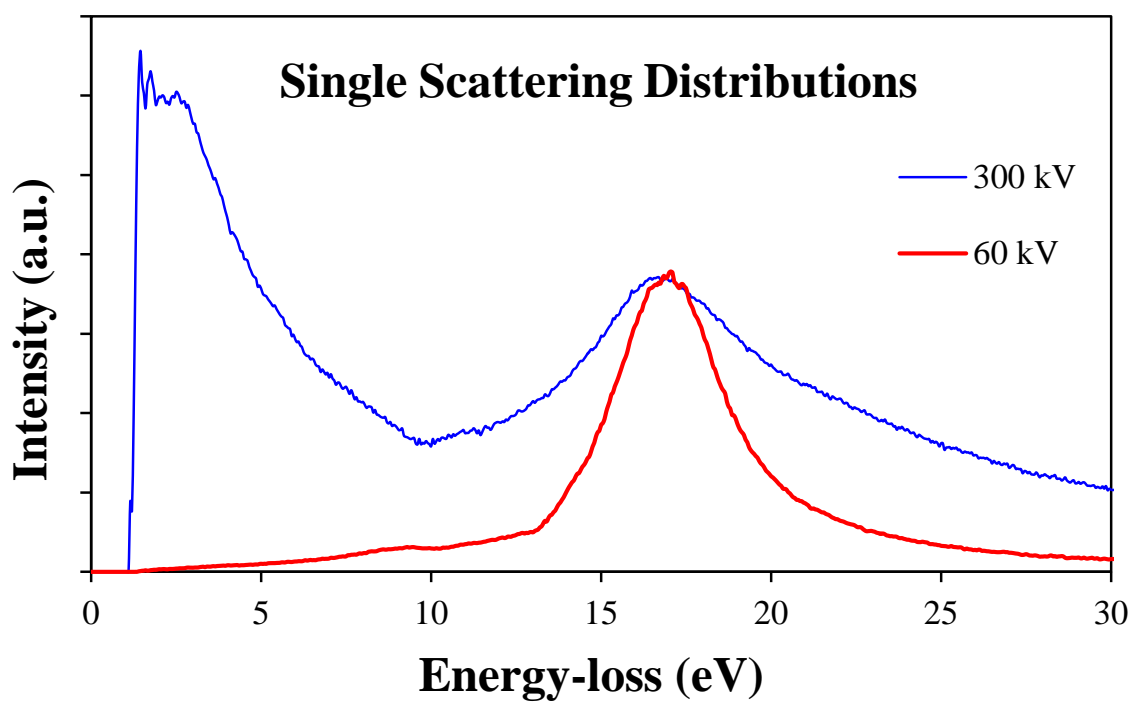

Figure 2. Single scattering distributions for $\mathrm{Si}$ at $300 \mathrm{kV}$ and $60 \mathrm{kV}$. The significant peak at $3 \mathrm{eV}$ can be seen at $300 \mathrm{kV}$, but is nearly completely removed at $60 \mathrm{kV}$. 